\title{
Anti-Streptococcus pyogenes Activity of Selected Medicinal Plant Extracts Used in Thai Traditional Medicine
}

\author{
Surasak Limsuwan ${ }^{1}$ and Supayang $\mathbf{P}$ Voravuthikunchai ${ }^{2^{\star}}$ \\ ${ }^{1}$ Faculty of Traditional Thai Medicine and Natural Products Research Center of Excellence, ${ }^{2}$ Department of Microbiology and \\ Natural Products Research Center of Excellence, Faculty of Science, Prince of Songkla University, Hat Yai, Songkhla, Thailand
}

*For correspondence: Email: supayang.v@psu.ac.th; Tel: +6674 446661

\begin{abstract}
Purpose: To evaluate the anti-Streptococcus pyogenes activity of selected medicinal plants used in Thai traditional medicine.

Methods: Sixty-nine extracts of 51 selected Thai medicinal plant species were tested for anti-S. pyogenes activity by paper disc agar diffusion and broth microdilution methods.

Results: Ten plants including Boesenbergia pandurata (Roxb.) Schltr., Cinnamomum bejolghota (Buch.-Ham.) Sweet, Cinnamomum porrectum (Roxb) Kosterm, Eleutherine americana Merr., Gymnopetalum cochinchinensis (Lour.) Kurz, Piper betle L., Quercus infectoria G. Olivier, Quisqualis indica L, Rhodomyrtus tomentosa (Aiton) Hassk., and Walsura robusta Roxb. demonstrated good antibacterial activity against S. pyogenes NPRC 101. These plants were selected and further evaluated for their anti-S. pyogenes activity against 11 isolates of $S$. pyogenes from patients with upper respiratory tract infections. Three plants including Boesenbergia pandurata, Eleutherine americana, and Rhodomyrtus tomentosa exhibited good antibacterial activity against all $S$. pyogenes isolates and produced similar activities against different tested isolates. Boesenbergia pandurata and Rhodomyrtus tomentosa demonstrated antibacterial activity with the same minimal inhibitory concentration (MIC) and minimal bactericidal concentration (MBC) range of $3.91-31.25 \mu \mathrm{g} / \mathrm{ml}$ whereas Eleutherine americana displayed MIC and MBC values of 250 and $250-500 \mu \mathrm{g} / \mathrm{ml}$ against all S. pyogenes isolates.

Conclusion: Boesenbergia pandurata, Eleutherine americana, and Rhodomyrtus tomentosa have great antibacterial potentials against $S$. pyogenes.
\end{abstract}

Keywords: Antibacterial activity, Boesenbergia pandurata, Eleutherine americana, Rhodomyrtus tomentosa, Streptococcus pyogenes, Thai medicinal plant, Upper respiratory tract infections

Tropical Journal of Pharmaceutical Research is indexed by Science Citation Index (SciSearch), Scopus, International Pharmaceutical Abstract, Chemical Abstracts, Embase, Index Copernicus, EBSCO, African Index Medicus, JournalSeek, Journal Citation Reports/Science Edition, Directory of Open Access Journals (DOAJ), African Journal Online, Bioline International, Open-J-Gate and Pharmacy Abstracts

\section{INTRODUCTION}

Upper respiratory tract infections (URTIs) are the most common human infection, mostly caused by viruses and bacteria. Streptococcus pyogenes is a major upper respiratory tract bacterial pathogen that causes a wide variety of diseases. It is the most common cause of bacterial pharyngitis and is linked to many serious complications [1]. Viral infections in upper respiratory tract are usually not an appropriate indication for the use of antibiotics. Bacteria that cause URTIs should be taken and cultured to determine the particular type of bacteria and antibiotics treatment are performed if necessary [2]. However, unnecessary and irrational selfmedication with antibiotics seems to be common for URTIs [3,4] and these result in resistance to many bacteria including $S$. pyogenes $[5,6]$. Antibiotic misuse for URTIs is a serious problem that not only results in selection of resistant strains of bacteria but also waste of resources. Correlation of antibiotic resistance in $S$. 
pyogenes with antibiotic consumption has been recorded. Increase in antibiotic use has resulted in increased prevalence of antibiotic-resistant $S$. pyogenes $[7,8]$. Hence, the search for alternative treatment dealing with URTIs caused by $S$. pyogenes is necessary. Medicinal plants are a great source of alternative treatment for many infections. The use of medicinal plants may substitute antibiotic consumption for URTIs or decrease antibiotic-resistant bacteria.

In traditional Thai medicine, many medicinal plants have been in use since ancient times. Herbal medicines are relatively safer than synthetic drugs and offer profound therapeutic benefits [9]. A number of Thai medicinal plants have been studied for their antibacterial activities. There are several reports on antibacterial activity of plants that inhibit various bacterial pathogens, but only limited number of studies on $S$. pyogenes, an important bacterial human pathogen, have been published. Therefore, this study was aimed to evaluate the antibacterial activity of selected medicinal plants commonly used in traditional Thai medicine for bacterial infections against $S$. pyogenes isolated from upper respiratory tract infections.

\section{EXPERIMENTAL}

\section{Preparation of plant extract}

Fifty-one medicinal plant species used in traditional Thai medicine for bacterial infections were selected. The plant materials were collected from various areas of the southern region of Thailand from 2006 - 2007. Quercus infectoria nut galls were purchased from an herb shop in Songkhla, Thailand. Botanical identification was performed by Dr. Oratai Neamsuvan, an ethnobotanist at the Faculty of Traditional Thai Medicine, Prince of Songkla University, where their voucher specimens are deposited. All plant materials were cut into small pieces and dried at $60{ }^{\circ} \mathrm{C}$ overnight. The dried plant materials were crushed in a mechanical mortar and soaked in extracted solvent for 7 days (3 times). The solvent was then filtered and dried using a rotary evaporator. All extracts were stored at $-4{ }^{\circ} \mathrm{C}$, and dissolved in dimethyl sulfoxide (DMSO, Merck, Germany) before use. The aliquots were checked for sterility by streaking with a sterile loop on brain heart infusion (BHI) agar and incubating at $37{ }^{\circ} \mathrm{C}$ overnight.

\section{Bacterial strain and culture conditions}

Eleven clinical isolates of $S$. pyogenes (NPRC 101-111) from patients with tonsillitis or pharyngitis were obtained from Department of Microbiology and Natural Products Research Center, Faculty of Science, Prince of Songkla University. All isolates were susceptible to erythromycin and penicillin $\mathrm{G}$. These isolates were stored in $\mathrm{BHI}$ broth supplement with $20 \%$ glycerol at $-70^{\circ} \mathrm{C}$. All the isolates were routinely cultured in $\mathrm{BHI}$ broth or blood agar (BA) plates and incubated with $5 \% \mathrm{CO}_{2}$ at $37^{\circ} \mathrm{C}$ for $24 \mathrm{~h}$.

\section{Screening for anti-S. pyogenes activity}

The preliminary screening of all plant extracts for their anti-S. pyogenes activity was carried out by disc agar diffusion method [10]. The extracts were dissolved in DMSO $(250 \mathrm{mg} / \mathrm{ml})$ and then $10 \mu \mathrm{l}$ were applied to sterile filter paper discs (Whatman No. 1; $6 \mathrm{~mm}$ in diameter) so that each disc finally yielded $2.5 \mathrm{mg}$ of the extract. Dry discs (dried at $37{ }^{\circ} \mathrm{C}$ overnight) were applied onto the surface of $5 \%$ blood Mueller Hinton agar (MHA) plates seeded with the culture of $S$. pyogenes. The plates were then incubated with $5 \% \mathrm{CO}_{2}$ at $37{ }^{\circ} \mathrm{C}$ for $20 \mathrm{~h}$. Dimethyl sulphoxide, extraction solvents, and antibiotic discs including erythromycin $(15 \mu \mathrm{g})$ and penicillin $\mathrm{G}(1 \mu \mathrm{g})$ were used as controls. The experiment was carried out in duplicate and the average diameter of inhibition zone was calculated.

\section{Determination of minimal inhibitory concentration (MIC) and minimal bactericidal concentration (MBC)}

A broth microdilution method according to Clinical and Laboratory Standards Institute Guidelines (CLSI) was used to determine the MIC and MBC of the plant extracts against $S$. pyogenes [10]. Erythromycin was used as reference antibiotic. MIC was recorded as the lowest concentration that produced a complete suppression of visible growth. MBC was taken as the concentration that gave significant MIC values using a sterile loop streaking on fresh media. All assays were carried out in triplicate.

\section{RESULTS}

\section{Screening for anti-S. pyogenes activity}

The antibacterial activities of 51 medicinal plant species used in Thai traditional medicine for bacterial infections against $S$. pyogenes NPRC 101 are presented in Table 1. Among the plants tested, nearly all extracts, except Murdannia loriformis, produced inhibition zones on $S$. pyogenes NPRC 101. The inhibition zones ranged from $7-26 \mathrm{~mm}$. Ethanol extracts of Piper betle, Coriandrum sativum, Quercus infectoria, and Eleutherine americana demonstrated large 
zones with diameters of $26,24,23$, and $23 \mathrm{~mm}$, respectively. Thirty four extracts (26 plant species) from a total of 69 extracts (51 plant species) possessed MIC values $\leq 1000 \mu \mathrm{g} / \mathrm{ml}$ on $S$. pyogenes NPRC 101 . Only 22 extracts (17 plant species) exhibited bactericidal activity at
MBC values $\leq 1000 \mu \mathrm{g} / \mathrm{ml}$. Among the plant species tested, Rhodomyrtus tomentosa (flower, fruit, and leaf extracts) and Boesenbergia pandurata (rhizome extract) produced better activity against $S$. pyogenes NPRC 101, as indicated by lower MIC and MBC values.

Table 1: Antibacterial activity of selected Thai medicinal plants against Streptococcus pyogenes NPRC 101

\begin{tabular}{|c|c|c|c|c|c|c|}
\hline Botanical species & Family & Voucher no. & $\begin{array}{l}\text { Plant } \\
\text { part }\end{array}$ & $\begin{array}{c}\text { Extract } \\
\text { yield (\%) }\end{array}$ & $\begin{array}{l}\text { Inhibi- } \\
\text { tion } \\
\text { zone }^{a} \\
(\mathrm{~mm})\end{array}$ & $\begin{array}{l}\mathrm{MIC} / \mathrm{MBC} \\
(\mu \mathrm{g} / \mathrm{ml})\end{array}$ \\
\hline Acacia catechu (L.f.) Willd. & Fabaceae & NPRCP0001 & core & $5.60 \mathrm{e}$ & 11 & $>1000 />1000$ \\
\hline Aegle marmelos (L.) Correa & Rutaceae & NPRCP0002 & fruit & $5.37 e$ & 15 & $>1000 />1000$ \\
\hline Alstonia scholaris (L.) R. Br. & Apocynaceae & NPRCP0003 & wood & $1.30 \mathrm{a}$ & 8 & $>1000 />1000$ \\
\hline Ardisia colorata Roxb. & Myrsinaceae & NPRCP0004 & fruit & $\begin{array}{l}5.60 \mathrm{a} \\
4.40 \mathrm{e}\end{array}$ & $\begin{array}{r}11 \\
9\end{array}$ & $\begin{array}{l}>1000 />1000 \\
>1000 />1000\end{array}$ \\
\hline Asclepias curassavica L. & Asclepiadaceae & NPRCP0005 & wood & $0.98 \mathrm{e}$ & 14 & $>1000 />1000$ \\
\hline $\begin{array}{l}\text { Boesenbergia pandurata (Roxb.) } \\
\text { Schltr. }\end{array}$ & Zingiberaceae & NPRCP0006 & rhizome & $1.58 \mathrm{c}$ & 7 & $7.81 / 7.81$ \\
\hline Cassia alata L. & Fabaceae & NPRCP0007 & leaf & $4.20 \mathrm{a}$ & 9 & $>1000 />1000$ \\
\hline Centella asiatica (L.) Urb. & Apiaceae & NPRCP0008 & leaf & $6.00 \mathrm{e}$ & 15 & $>1000 />1000$ \\
\hline Cinnamomum bejolghota (Buch.-Ham.) & Lauraceae & NPRCP0009 & bark & $14.68 \mathrm{e}$ & 19 & $250 / 250$ \\
\hline Sweet & & & wood & $2.29 \mathrm{e}$ & 16 & $125 / 125$ \\
\hline Cinnamomum porrectum (Roxb.) & Lauraceae & NPRCP0010 & bark & $7.09 \mathrm{e}$ & 19 & $500 / 1000$ \\
\hline Kosterm. & & & wood & $11.23 e$ & 16 & $250 / 250$ \\
\hline Cleome gynandra L. & Capparaceae & NPRCP0011 & $\begin{array}{l}\text { whole } \\
\text { plant }\end{array}$ & $6.76 \mathrm{e}$ & 11 & $1000 />1000$ \\
\hline Coriandrum sativum $\mathrm{L}$. & Apiaceae & NPRCP0012 & fruit & $\begin{array}{l}2.00 \mathrm{a} \\
4.00 \mathrm{e}\end{array}$ & $\begin{array}{l}16 \\
24\end{array}$ & $\begin{array}{r}>1000 />1000 \\
62.50 / 125\end{array}$ \\
\hline Curcuma zedoaria (Christm.) Roscoe & Zingiberaceae & NPRCP0013 & rhizome & $9.60 \mathrm{e}$ & 11 & $500 / 500$ \\
\hline Derris scandens Roxb. Benth. & Leguminosae & NPRCP0014 & stem & $\begin{array}{r}11.40 \mathrm{a} \\
3.20 \mathrm{e}\end{array}$ & $\begin{array}{l}13 \\
13\end{array}$ & $\begin{array}{l}125 / 125 \\
250 / 250\end{array}$ \\
\hline Dracaena loureoiri Gangnep. & Agavaceae & NPRCP0015 & core & $16.90 \mathrm{e}$ & 15 & $1000 />1000$ \\
\hline Dryopteris syrmatica O. Kze. & Polypodiaceae & NPRCP0016 & wood & $\begin{array}{l}4.50 \mathrm{a} \\
4.50 \mathrm{e}\end{array}$ & $\begin{array}{r}8 \\
13\end{array}$ & $\begin{array}{r}>1000 />1000 \\
125 / 125\end{array}$ \\
\hline Eleutherine americana Merr. & Iridaceae & NPRCP0017 & bulb & $4.80 \mathrm{e}$ & 23 & $250 / 500$ \\
\hline Euphorbia thymifolia L. & Euphorbiaceae & NPRCP0018 & $\begin{array}{l}\text { whole } \\
\text { plant }\end{array}$ & $1.30 \mathrm{e}$ & 15 & $500 />1000$ \\
\hline $\begin{array}{l}\text { Gymnopetalum cochinchinensis (Lour.) } \\
\text { Kurz }\end{array}$ & Cucurbitaceae & NPRCP0019 & fruit & $7.66 \mathrm{e}$ & 15 & $250 / 500$ \\
\hline $\begin{array}{l}\text { Holarrhena antidysenterica (L.) Wall. } \\
\text { ex A. DC. }\end{array}$ & Apocynaceae & NPRCP0020 & bark & $2.10 \mathrm{e}$ & 15 & $500 / 500$ \\
\hline Impatiens balsamina L. & Balsaminaceae & NPRCP0021 & leaf & $5.20 \mathrm{e}$ & 15 & $>1000 />1000$ \\
\hline Manilkara achras (Mill.) Fosberg & Sapotaceae & NPRCP0022 & fruit & $26.77 e$ & 9 & $>1000 />1000$ \\
\hline Millingtonia hortensis L. $\mathrm{f}$. & Bignoniaceae & NPRCP0023 & flower & $25.41 \mathrm{e}$ & 8 & $>1000 />1000$ \\
\hline Mimosa pudica L. & Fabaceae & NPRCP0024 & $\begin{array}{l}\text { whole } \\
\text { plant }\end{array}$ & $4.91 \mathrm{e}$ & 10 & $>1000 />1000$ \\
\hline Mitragyna speciosa (Korth.) Havil & Rubiaceae & NPRCP0025 & leaf & $5.96 e$ & 8 & $1000 />1000$ \\
\hline Momordica charantia L. & Cucurbitaceae & NPRCP0026 & vine & $3.00 \mathrm{e}$ & 15 & $1000 / 1000$ \\
\hline Morinda citrifolia L. & Rubiaceae & NPRCP0027 & fruit & $7.36 \mathrm{e}$ & 9 & $>1000 />1000$ \\
\hline $\begin{array}{l}\text { Murdannia loriformis (Hassk.) R.S. } \\
\text { Rao \& Kammathy }\end{array}$ & Commelinaceae & NPRCP0028 & $\begin{array}{l}\text { whole } \\
\text { plant }\end{array}$ & $7.67 e$ & $-b$ & $N A^{c}$ \\
\hline Oroxylum indicum (L.) Kurz & Bignoniaceae & NPRCP0029 & bark & $3.71 \mathrm{e}$ & 10 & $>1000 />1000$ \\
\hline $\begin{array}{l}\text { Peltophorum pterocarpum (DC.) } \\
\text { Backer ex K. Heyne }\end{array}$ & Fabaceae & NPRCP0030 & bark & $\begin{array}{c}0.03 \mathrm{~d} \\
0.01 \mathrm{~h} \\
6.20 \mathrm{~m}\end{array}$ & $\begin{array}{l}15 \\
12 \\
15\end{array}$ & $\begin{array}{r}1000 />1000 \\
>1000 />1000 \\
1000 />1000\end{array}$ \\
\hline $\begin{array}{l}\text { Phyllanthus amarus Schumach. \& } \\
\text { Thonn. }\end{array}$ & Euphorbiaceae & NPRCP0031 & $\begin{array}{l}\text { whole } \\
\text { plant }\end{array}$ & $7.82 \mathrm{e}$ & 10 & $>1000 />1000$ \\
\hline Piper betle L. & Piperaceae & NPRCP0032 & leaf & $9.19 \mathrm{e}$ & 26 & $500 / 500$ \\
\hline Piper chaba Hunter & Piperaceae & NPRCP0033 & fruit & $8.96 \mathrm{e}$ & 9 & $>1000 />1000$ \\
\hline Piper nigrum L. & Piperaceae & NPRCP0034 & fruit & $6.33 \mathrm{e}$ & 10 & $>1000 />1000$ \\
\hline Piper sarmentosum Roxb. & Piperaceae & NPRCP0035 & leaf & $4.72 \mathrm{e}$ & 20 & $>1000 />1000$ \\
\hline Pluchea indica (L.) Less. & Asteraceae & NPRCP0036 & leaf & $17.80 \mathrm{e}$ & 14 & $500 />1000$ \\
\hline Psidium guajava L. & Myrtaceae & NPRCP0037 & leaf & $2.80 a$ & 12 & $>1000 />1000$ \\
\hline Quercus infectoria G. Olivier & Fagaceae & NPRCP0038 & nut gall & $57.15 \mathrm{e}$ & 23 & $500 / 1000$ \\
\hline Quisqualis indica L. & Combretaceae & NPRCP0039 & flower & $11.08 \mathrm{e}$ & 17 & $250 / 500$ \\
\hline Rhizophora mucronata Lam. & Rhizophoraceae & NPRCP0040 & $\begin{array}{l}\text { bark } \\
\text { fruit }\end{array}$ & $\begin{array}{l}11.67 \mathrm{e} \\
10.75 \mathrm{e}\end{array}$ & $\begin{array}{l}15 \\
20\end{array}$ & $\begin{array}{r}>1000 />1000 \\
1000 />1000\end{array}$ \\
\hline
\end{tabular}


Table 1 (contd.): Antibacterial activity of selected Thai medicinal plants against Streptococcus pyogenes NPRC 101

\begin{tabular}{|c|c|c|c|c|c|c|}
\hline Botanical species & Family & Voucher no. & $\begin{array}{l}\text { Plant } \\
\text { part }\end{array}$ & $\begin{array}{r}\text { Extract } \\
\text { yield }(\%)\end{array}$ & $\begin{array}{r}\text { Inhibiti } \\
\text { on } \\
\text { zonea } \\
(\mathrm{mm})\end{array}$ & $\begin{array}{r}\mathrm{MIC} / \mathrm{MBC} \\
(\mu \mathrm{g} / \mathrm{ml})\end{array}$ \\
\hline \multirow[t]{4}{*}{ Rhodomyrtus tomentosa (Aiton) Hassk. } & Myrtaceae & NPRCP0041 & flower & $5.63 \mathrm{e}$ & 17 & $15.6 / 62.50$ \\
\hline & & & fruit & $2.47 \mathrm{e}$ & 16 & $15.6 / 62.50$ \\
\hline & & & leaf & $7.40 \mathrm{e}$ & 18 & $7.81 / 62.50$ \\
\hline & & & stem & $7.17 \mathrm{e}$ & 10 & $1000 />1000$ \\
\hline \multirow[t]{2}{*}{ Sandoricum indicum Cav. } & Meliaceae & NPRCP0042 & root & $5.60 a$ & 11 & $500 / 500$ \\
\hline & & & & $4.00 \mathrm{e}$ & 15 & $>1000 />1000$ \\
\hline Tamarindus indica L. & Fabaceae & NPRCP0043 & leaf & $4.80 \mathrm{e}$ & 14 & $>1000 />1000$ \\
\hline Terminalia bellirica (Gaertn.) Roxb. & Combretaceae & NPRCP0044 & fruit & $14.88 \mathrm{e}$ & 15 & $>1000 />1000$ \\
\hline Terminalia chebula Retz. & Combretaceae & NPRCP0045 & fruit & $17.20 \mathrm{e}$ & 13 & $>1000 />1000$ \\
\hline Terminalia sp. & Combretaceae & NPRCP0046 & fruit & $23.90 \mathrm{e}$ & 17 & $>1000 />1000$ \\
\hline Theobroma cacao L. & Sterculiaceae & NPRCP0047 & pericarp & $3.67 \mathrm{e}$ & 9 & $>1000 />1000$ \\
\hline Uncaria gambir & Rubiaceae & NPRCP0048 & $\begin{array}{l}\text { leaf, } \\
\text { branch }\end{array}$ & $\begin{array}{l}65.40 a \\
65.40 \mathrm{e}\end{array}$ & $\begin{array}{l}11 \\
11\end{array}$ & $\begin{array}{r}>1000 />1000 \\
500 />1000\end{array}$ \\
\hline \multirow[t]{4}{*}{ Walsura robusta Roxb. } & Meliaceae & NPRCP0049 & leaf, & $0.59 a$ & 17 & $>1000 />1000$ \\
\hline & & & branch & $1.00 \mathrm{~b}$ & 9 & $1000 />1000$ \\
\hline & & & & 0.35 et & 16 & $125 / 500$ \\
\hline & & & & $12.09 \mathrm{~m}$ & 17 & $>1000 />1000$ \\
\hline $\begin{array}{l}\text { Wrightia tomentosa (Roxb.) Roem. \& } \\
\text { Schult. }\end{array}$ & Apocynaceae & NPRCP0050 & stem & $3.90 \mathrm{e}$ & 15 & $500 />1000$ \\
\hline \multirow[t]{2}{*}{ Xylocarpus granatum J. König } & Meliaceae & NPRCP0051 & pericarp & $2.68 \mathrm{e}$ & 10 & $>1000 />1000$ \\
\hline & & & seed & $6.77 \mathrm{e}$ & 11 & $>1000 />1000$ \\
\hline Erythromycin & & & & & & $0.125 / 0.125$ \\
\hline
\end{tabular}

Table 2: Minimum inhibitory concentration (MIC) and minimum bactericidal concentration (MBC) of 10 plant extracts against clinical Streptococcus pyogenes isolates $(n=11)$

\begin{tabular}{|c|c|c|c|}
\hline Botanical species & Part tested & MIC range $(\mu \mathrm{g} / \mathrm{ml})$ & MBC range $(\mu \mathrm{g} / \mathrm{ml})$ \\
\hline Boesenbergia pandurata $^{a}$ & rhizome & $3.91-31.25$ & $7.81-62.50$ \\
\hline Cinnamomum bejolghota $^{\mathrm{b}}$ & $\begin{array}{l}\text { bark } \\
\text { wood }\end{array}$ & $\begin{array}{r}31.25->1000 \\
31.25-1000\end{array}$ & $\begin{array}{r}31.25->1000 \\
31.25-1000\end{array}$ \\
\hline $\begin{array}{l}\text { Cinnamomum porrectum } \\
\text { Eleutherine americana }^{\mathrm{b}}\end{array}$ & $\begin{array}{l}\text { bark } \\
\text { wood } \\
\text { bulb }\end{array}$ & $\begin{array}{r}62.50->1000 \\
250->1000 \\
250\end{array}$ & $\begin{array}{r}62.50->1000 \\
250->1000 \\
250-500\end{array}$ \\
\hline Gymnopetalum cochinchinensis $^{\mathrm{b}}$ & fruit & $31.25->1000$ & $31.25->1000$ \\
\hline Piper betle ${ }^{\mathrm{b}}$ & leaf & $500-1000$ & $500-1000$ \\
\hline Quercus infectoria $^{\mathrm{b}}$ & nut gall & $125->1000$ & $125->1000$ \\
\hline Quisqualis indica $^{\mathrm{b}}$ & flower & $250->1000$ & $250->1000$ \\
\hline Rhodomyrtus tomentosa ${ }^{\mathrm{b}}$ & leaf & $3.91-31.25$ & $3.91-62.50$ \\
\hline Walsura robusta $^{\mathrm{c}}$ & leaf, branch & $62.50->1000$ & $62.50->1000$ \\
\hline Erythromycin & & $<0.015-0.125$ & $<0.015-0.125$ \\
\hline
\end{tabular}

${ }^{a}$ Chloroform extract; ${ }^{b}$ Ethanol extract; ${ }^{c}$ Ethyl acetate extract.

\section{Anti-S. pyogenes activity of plant extracts}

Ten effective plants, including Boesenbergia pandurata, Cinnamomum bejolghota, Cinnamomum porrectum, Eleutherine americana, Gymnopetalum cochinchinensis, Piper betle, Quercus infectoria, Quisqualis indica, Rhodomyrtus tomentosa, and Walsura robusta were selected based on their antibacterial activity against S. pyogenes NPRC 101 to further determine the variability of MICs and MBCs against 11 clinical isolates. The MIC and MBC values of these 10 effective plants against 11 clinical isolates of $S$. pyogenes are shown in Table 2. Variations in the MIC and MBC values were found among the bacterial isolates when tested with the extracts of Cinnamomum bejolghota, Cinnamomum porrectum, Gymnopetalum cochinchinensis, Quercus infectoria, Quisqualis indica, and Walsura robusta. These plants exhibited a wide range of MIC and MBC values, from 31.25 - >1000 and 
31.25 - >1000 $\mu \mathrm{g} / \mathrm{ml}$, respectively. In contrast, three plant species including Boesenbergia pandurata, Eleutherine americana, and Rhodomyrtus tomentosa showed similar antibacterial activity among different 11 clinical isolates. Boesenbergia pandurata and Rhodomyrtus tomentosa demonstrated very good antibacterial activity with MIC and MBC values ranged from $3.91-31.25,3.91-31.25$ and $7.81-62.50,3.91-62.50 \mu \mathrm{g} / \mathrm{ml}$, respectively. Meanwhile the extract of Eleutherine americana demonstrated moderate activity against all isolates with the MIC and MBC values of 250 and $250-500 \mu \mathrm{g} / \mathrm{ml}$, respectively. All isolates tested were sensitive to erythromycin (MIC $\leq 0.25 \mu \mathrm{g} / \mathrm{ml}$ ).

\section{DISCUSSION}

A number of plant extracts can be screened for their anti-S. pyogenes properties quickly using paper disc assay. However, this assay is not classically quantitative and using the size of inhibition zone to indicate relative antibacterial activity is not sufficient. The zone of inhibition may be affected by many factors such as the evaporation, solubility, and diffusion rate of the active components through test medium. Zone of inhibition testing is particularly appropriate for determining the ability of water-soluble antimicrobial compounds to inhibit the growth of microorganisms. Therefore, the MIC/MBC values and zone of inhibition of some of the plant extracts in this study did not correlate. For example, Boesenbergia pandurata extract generated a very small zone of inhibition $(7 \mathrm{~mm})$ but possessed very good MIC and MBC values.

In this study, we found that the extracts of Boesenbergia pandurata, Eleutherine americana, and Rhodomyrtus tomentosa not only demonstrated good antibacterial activity against $S$. pyogenes isolated from upper respiratory tract infections but also produced similar activities against different $S$. pyogenes clinical isolates.

In Thai traditional medicine, the rhizomes of Boesenbergia pandurata are used to treat colic disorders, wound infections and inflammation [11]. The antibacterial activities of this plant have been reported [12]. Some antibacterial active components from this plant were isolated and studied for their activities. Pinostrobin isolated from this plant demonstrated anti-Helicobacter pylori activity [13]. Panduratin A from this plant displayed significant antibacterial activity against a number of staphylococci and enterococci clinical isolates. Notably, the antibacterial activity of panduratin $A$ was more potent than many reference antibiotics [14,15]. Isopanduratin A from this plant demonstrated antibacterial activity against many streptococci including Streptococcus mutans, Streptococcus sobrinus, Streptococcus sanguinis, and Streptococcus salivarius $[16,17]$.

Eleutherine americana is a herbal plant whose red bulb was used as a folk medicine and flavouring agent. In the screening test we found that the bulb extract of this plant demonstrated moderately strong activity against $S$. pyogenes when compared with other plant extracts. However, when we increased the number of bacterial isolates it could produce similar antibacterial activity against difference $S$. pyogenes isolates. The antibacterial activities of this plant have been previously reported against gram-negative [18] and gram-positive bacteria [19]. Several compounds from this plant such as eleuthinone $A$, eleuthraquinone $A$ and $B$, and eleucanarol have been isolated and studied for their antibacterial activities against Staphylococcus aureus [20].

Rhodomyrtus tomentosa is a Thai medicinal plant used to treat skin, oral, gastrointestinal, and urinary tract infections. In this study, we found that the extracts of flower, fruit, and leaf demonstrated good antibacterial activity against S. pyogenes. The leaf extract of this plant has been reported for its antibacterial activity against many bacterial pathogens [19]. An isolated compound, named rhodomyrtone, from the leaf of this plant exhibited strong antibacterial activity against many pathogenic Gram-positive bacteria [21-23] including $S$. pyogenes [24]. Moreover, rhodomyrtone possessed noteworthy activity against methicillin-resistant $S$. aureus, displaying a stronger activity than vancomycin, a reference antibiotic [24].

\section{CONCLUSION}

Our study demonstrated that the plants species including Boesenbergia pandurata, Eleutherine americana, and Rhodomyrtus tomentosa have great potentials as antibacterial agents against $S$. pyogenes. Thus, these plants may yield biologically active compounds that might be valuable in the treatment of the diseases caused by $S$. pyogenes. Their active components, however, need to be isolated, and their toxicity and therapeutic activity in vivo evaluated.

\section{ACKNOWLEDGMENT}

This work was funded by the Thailand Research Fund (BRG 5580015. We thank Dr. Oratai Neamsuvan for her help on plant identification. 


\section{REFERENCES}

1. Cunningham MW. Pathogenesis of group $A$ streptococcal infections. Clin Microbiol Rev 2000; 13: 470-511.

2. Summers A. Sore throats. Accid Emerg Nurs 2005; 13 : 15-17.

3. Vaananen $M H$, Pietila K, Airaksinen M. Self-medication with antibiotics-Does it really happen in Europe? Health Policy 2006; 77: 166-171.

4. Grigoryan L, Burgerhof JG, Haaijer-Ruskamp FM, Degener JE, Deschepper R, Monnet DL, $D$ Matteo A, Scicluna EA, Bara AC, Lundborg CS, et al. Is self-medication with antibiotics in Europe driven by prescribed use? J Antimicrob Chemother 2007; 59: 152-156.

5. Malli E, Tatsidou E, Damani A, Pantelidi K, Petinaki E, Skoulakis C, Drougka E, Spiliopoulou I. Macrolide-resistant Streptococcus pyogenes in central Greece: prevalence; mechanism and molecular identification. Int J Antimicrob Agents 2010; 35: 614-615.

6. Vranes J, Knezevic J, Bedenic B, Stimac D, JarzaDavila $N$, Anusic $M$. The relationship between macrolide resistance in Streptococcus pneumoniae and consumption of oral macrolides in Republic of Croatia and City of Zagreb. Int $J$ Infect Dis 2010; 14: e343-e344.

7. Bergman $M$, Huikko S, Pihlajamaki $M$, Laippala $P$, Palva $E$, Huovinen $P$, Seppala $H$. Effect of macrolide consumption on erythromycin resistance in Streptococcus pyogenes in Finland in 19972001. Clin Infect Dis 2004; 38: 1251-1256.

8. Hsueh PR, Shyr JM, Wu JJ. Decreased erythromycin use after antimicrobial reimbursement restriction for undocumented bacterial upper respiratory tract infections significantly reduced erythromycin resistance in Streptococcus pyogenes in Taiwan. Clin Infect Dis 2005; 40: 903-905.

9. Barrett $B$, Kiefer D, Rabago D. Assessing the risks and benefits of herbal medicine: an overview of scientific evidence. Altern Ther Health Med 1999; 5: 40-49.

10. Clinical and Laboratory Standards Institute: CLSI. Methods for dilution antimicrobial susceptibility tests for bacteria that grow aerobically; Approved standard, $8^{\text {th }}$ ed. Clinical and Laboratory Standards Institute document M07-A8. Wayne, $P A$, Laboratory Standards Institute, 2009.

11. Voravuthikunchai $S P$, Lmsuwan $S$, Chusri $S$. New perspectives on herbal medicines for bacterial infection: Natural products II. In: Govil JN, Singh VK, Siddqui NT, editors. Recent progress in medicinal plants. Vol. 18. USA: Studium Press, LLC; 2007. p. 41-101.

12. Voravuthikunchai SP, Limsuwan $S$, Supapol $O$, Subhadhirasakul S. Antibacterial activity of extracts from family Zingiberaceae against foodborne pathogens. J Food Saf 2006; 26: 325334.

13. Bhamarapravati S, Juthapruth S, Mahachai W, Mahady G. Antibacterial activity of Boesenbergia rotunda (L.) Mansf. and Myristica fragrans Houtt. against Helicobacter pylori. Songklanakarin J Sci Technol 2006; 28: 157-163.

14. Rukayadi Y, Lee $K$, Han S, Yong $D$, Hwang JK. In vitro activities of panduratin $A$ against clinical Staphylococcus strains. Antimicrob Agents Chemother 2009; 53: 4529-4532.

15. Rukayadi Y, Han S, Yong D, Hwang JK. In Vitro antibacterial activity of panduratin $A$ against enterococci clinical isolates. Biol Pharm Bull 2010; 33: 1489-1493.

16. Hwang JK, Chung JY, Baek NI, Park JH. Isopanduratin $A$ from Kaempferia pandurata as an active antibacterial agent against cariogenic Streptococcus mutans. Int J Antimicrob Agents 2004; 23: 377-381.

17. Hwang JK, Shim JS, Chung JY. Anticariogenic activity of some tropical medicinal plants against Streptococcus mutans. Fitoterapia 2004; 75: 596-598.

18. Sirirak T, Voravuthikunchai SP. Eleutherine americana: A Candidate for the control of Campylobacter species. Poult Sci 2011; 90: 791-796.

19. Limsuwan S, Subhadhirasakul S, Voravuthikunchai SP. Medicinal plants with significant activity against important pathogenic bacteria. Pharm Biol 2009; 47: 683-689.

20. Mahabusarakam W, Hemtasin C, Chakthong S, Voravuthikunchai SP, Olawumi IB. Naphthoquinones, anthraquinones and naphthalene derivatives from the bulbs of Eleutherine americana. Planta Med 2010; 76: 345-349.

21. Sianglum $W$, Srimanote $P$, Wonglumsom $W$, Kittiniyom K, Voravuthikunchai SP. Proteome analyses of cellular proteins in methicillin-resistant Staphylococcus aureus treated with rhodomyrtone, a novel antibiotic candidate. PLoS One 2011; 6: e16628.

22. Visutthi $M$, Srimanote $P$, Voravuthikunchai $S P$. Responses in the expression of extracellular proteins in methicillin-resistant Staphylococcus aureus treated with rhodomyrtone. J Microbiol 2011; 49: 956-964.

23. Saising J, Voravuthikunchai SP. Anti Propionibacterium acnes activity of rhodomyrtone, an effective compound from Rhodomyrtus tomentosa (Aiton) Hassk. leaves. Anaerobe 2012; 18: 400-404.

24. Limsuwan S, Trip EN, Kouwen TRHM, Piersma S, Hiranrat $A$, Mahabusarakam $W$, Voravuthikunchai SP, van Dijl JM, Kayser O. Rhodomyrtone: A new candidate as natural antibacterial drug from Rhodomyrtus tomentosa. Phytomedicine 2009; 16: 645-651. 